\title{
Design of Surfactant-Substrate Interactions for Roll-to-Roll Assembly of Carbon Nanotubes for Thin-Film Transistors
}

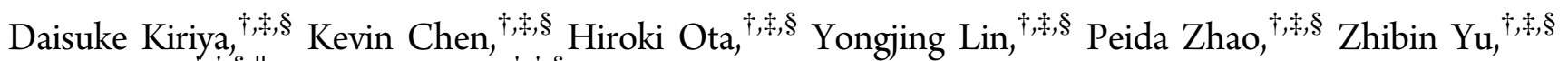 \\ Tae-jun $\mathrm{Ha},{ }^{\dagger, \$, \S, \|}$ and Ali Javey ${ }^{*, \dagger, \ddagger, \S}$
}

${ }^{\dagger}$ Electrical Engineering and Computer Sciences and ${ }^{\S}$ Berkeley Sensor and Actuator Center, University of California at Berkeley, Berkeley, California 94720, United States

${ }^{\ddagger}$ Materials Sciences Division, Lawrence Berkeley National Laboratory, Berkeley, California 94720, United States

\section{Supporting Information}

ABSTRACT: Controlled assembly of single-walled carbon nanotube (SWCNT) networks with high density and deposition rate is critical for many practical applications, including large-area electronics. In this regard, surfactant chemistry plays a critical role as it facilitates the substratenanotube interactions. Despite its importance, detailed understanding of the subject up until now has been lacking, especially toward tuning the controllability of SWCNT assembly for thin-film transistors. Here, we explore SWCNT assembly with steroid- and alkyl-based surfactants. While steroid-based surfactants yield highly dense nanotube thin

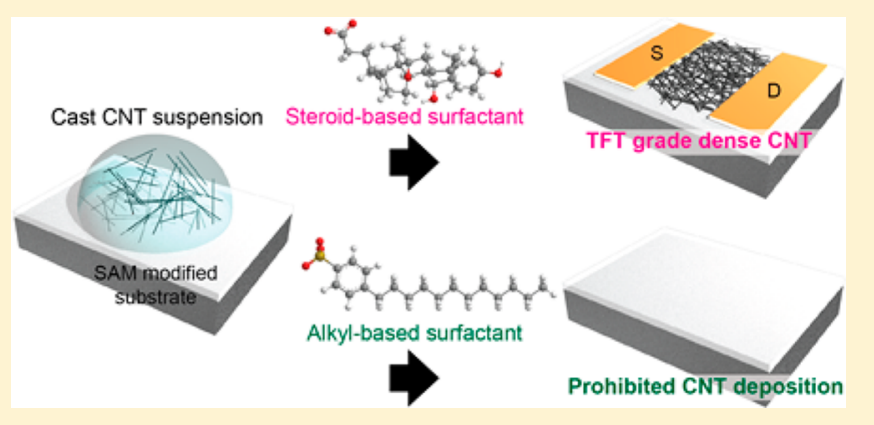
films, alkyl surfactants are found to prohibit nanotube assembly. The latter is attributed to the formation of packed alkyl layers of residual surfactants on the substrate surface, which subsequently repel surfactant encapsulated SWCNTs. In addition, temperature is found to enhance the nanotube deposition rate and density. Using this knowledge, we demonstrate highly dense and rapid assembly with an effective SWCNT surface coverage of $\sim 99 \%$ as characterized by capacitance-voltage measurements. The scalability of the process is demonstrated through a roll-to-roll assembly of SWCNTs on plastic substrates for large-area thin-film transistors. The work presents an important process scheme for nanomanufacturing of SWCNT-based electronics.

\section{INTRODUCTION}

Single-walled carbon nanotubes (SWCNTs) exhibit unique physical $^{1,2}$ and chemical $^{3}$ properties, including high carrier mobility, ${ }^{4}$ excellent chemical ${ }^{5}$ and mechanical ${ }^{6,7}$ robustness, and intriguing photothermal effects. ${ }^{8,9}$ These characteristics have triggered the exploration of SWCNTs toward a wide range of new applications; ${ }^{10-20}$ one example being the use of SWCNT random networks as the active channel material for thin-film transistors (TFTs). ${ }^{21,22}$ Given recent advancements in electronic-type and chirality purification of SWCNTs, ${ }^{23-25}$ random networks of SWCNTs can be solution deposited on various flexible $\mathrm{e}^{11,21,26-28}$ and rigid substrates ${ }^{11,21,29-32}$ and configured as high-performance TFTs. These TFTs have been utilized as the active matrix backplane for organic light-emitting diode displays ${ }^{33}$ and user-interactive surfaces, ${ }^{34}$ demonstrating their utility for large-area electronic systems. Uniquely, gravure $^{35,36}$ or inkjet ${ }^{37}$ printed TFTs based on SWCNT networks have also been reported, demonstrating excellent performances, surpassing that of printed organic devices by a large margin. The performance of SWCNT TFTs is largely dependent on the properties of the assembled random networks. ${ }^{38}$ Specifically, high nanotube density yields high ON-state current, while bundling during assembly increases the OFF-state current. ${ }^{39,40}$ Thus, understanding and controlling the nanotube-substrate binding as facilitated through the surfactant-surface interactions is of profound interest, with strong emphasis on enhancing density and reducing bundling. Furthermore, fast nanotube assembly on substrates is essential for practical applications as it determines the process throughput, eventually allowing roll-to-roll (R2R) assembly and processing of SWCNTs over large-areas.

Significant research has focused on dispersion of SWCNTs in liquids over the past decade, ${ }^{41-60}$ with one promising method involving the use of aqueous solutions with organic surfactants that show long-term stability over several months. ${ }^{52,54,55}$ Thin and thick films of SWCNTs from nanotube suspensions have also been widely explored for various applications. $9,15,33,34,36,61$ However, the details of the assembly process of SWCNTs for "TFT-grade" random networks have not been fully explored, which is a critical step for optimizing the deposition quality and rate. In addition, most work in literature has focused on the use of commercially available solutions of semiconductor-enriched SWCNTs for TFT fabrication. The surfactants used for these commercial solutions are often not publically known, and a change in the surfactant composition by the manufacturer can often drastically affect the nanotube deposition quality, without the knowledge of the researchers. These shortcomings present

Received: June 24, 2014

Published: July 14, 2014 
(a)

(b)

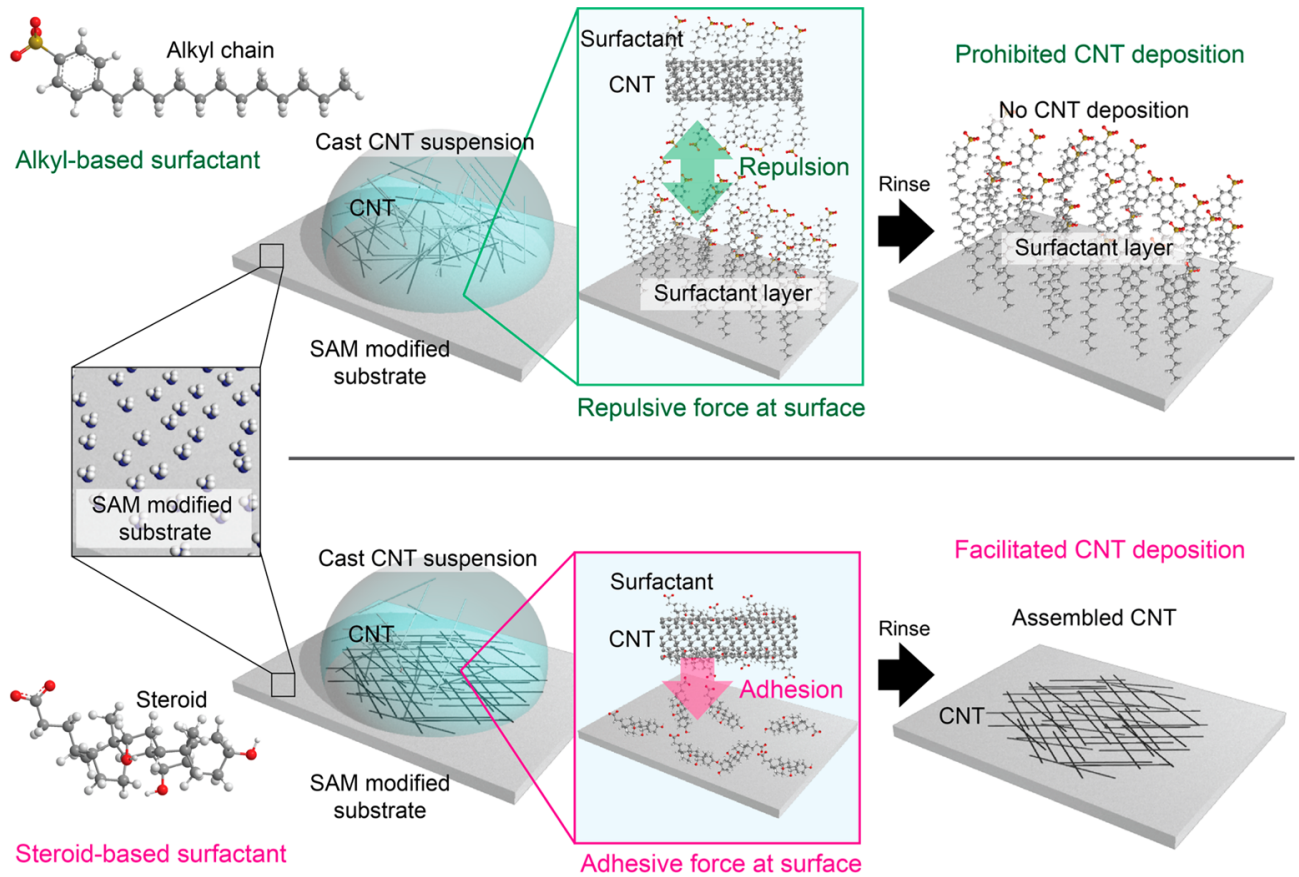

Figure 1. Schematic illustration of the solution-based SWCNT assembly process. (a) Deposition of a SWCNT solution with an alkyl-type surfactant (AS). The illustrated chemical is SDBS. The residual AS is assembled onto the surface of the amine-modified (self-assembled monolayer: SAM) substrate which induce a repulsive force against SWCNTs wrapped with the same surfactant. Thus, SWCNTs are not assembled, and only surfactants remain on the surface of the substrate. (b) Deposition process from a suspension with a steroid type surfactant (SS). Because of the rigid structure of the steroid surfactants, a dense blocking layer shown in AS is not formed on the substrate, thus facilitating adhesion of SWCNTs.

fundamental challenges to the scientific community in advancing the use of SWCNT TFTs for large-area systems. In this work, we systematically explore the effect of surfactant chemistry on nanotube assembly for amine functionalized surfaces. Starting from commercially available 99\% semiconducting SWCNT powders, we demonstrate drastic differences in the nanotube assembly for alkyl- and steroid-based surfactants, with the former yielding almost no assembly and the latter resulting in high-density networks. We explore the underlying mechanism for the assembly as mediated by the surfactant. By engineering the various chemical interactions in this system, we demonstrate high-density SWCNT networks with effective surface coverage of up to $\sim 99 \%$ as confirmed by capacitance-voltage measurements. Furthermore, we show that the assembly rate can be drastically enhanced by increasing solution temperature, thus providing a high-throughput process scheme. As an example, we demonstrate a R2R process for assembling SWCNT networks on a flexible substrate over $1 \mathrm{~m}$ in length. This work presents an important advance toward understanding and engineering nanotube assembly over large areas for use in TFT applications.

\section{RESULTS AND DISCUSSION}

The choice of surfactant used for the SWCNT solution has a drastic effect on the nanotube assembly process attributed to multiple factors in the system. Specifically, residual surfactants (i.e., extra surfactants that do not encapsulate the SWCNTs) that always exist in the solution can block off the amine adhesion sites of the substrate and prevent nanotube assembly. ${ }^{62}$ In this regard, the surfactant must be wisely selected when considering TFT applications. Figure 1 represents a schematic illustration of the assembly process from SWCNT solutions with two types of surfactants, alkylchain-based surfactants (AS) and steroid-based surfactants (SS) used in this work. After casting the solution onto aminemodified substrates, residual AS in the solution immediately self-assembles into a packed surfactant layer on the substrate. This packed AS layer prohibits the assembly of AS-coated SWCNTs due to repulsive electrostatic forces (Figure 1a). ${ }^{63}$ On the other hand, SS allows for the dense assembly of SWCNTs because residual SS cannot form into a packed layer as shown in AS on amine functionalized surfaces (Figure 1b). ${ }^{64}$ The rigid nature of the steroid moiety can be attributed as the main reason for its loosely packed structure on the amine surface, in comparison to alkyl moiety's more flexible and therefore tight packing assembled structure.

In this research, we targeted commonly used surfactants such as sodium sulfate (SDS), sodium dodecylbenzenesulfonate (SDBS), and sodium dodecanoate (SDC) as AS and sodium cholate (SC) and sodium deoxy cholate (SDeC) as SS. 99\% semiconducting SWCNT powder (Nanointegris Inc.) was suspended in the various surfactant solutions mentioned above $(\sim 0.01 \mathrm{mg} \mathrm{SWCNT} / \mathrm{mL}, \sim 30 \mu \mathrm{mol}$ surfactant $/ \mathrm{ml})$ via probe sonication $(20 \mathrm{kHz}, 150 \mathrm{~W}$ ) (Figure $2 \mathrm{a}$ and Experimental Section). All solutions show absorption peaks around $450 \mathrm{~nm}$ corresponding to van Hove transitions, ${ }^{65}$ indicating isolated SWCNTs in the solutions. Interestingly, although all surfactant suspensions show similar optical characteristics, the assembling properties of SWCNTs onto a poly-L-lysine (PLL) modified $\mathrm{SiO}_{2} / \mathrm{Si}$ substrate are drastically different (Figures $2 \mathrm{c}$ and $\mathrm{S} 1$ ). In the cases of SDBS, SDS, and SDC, no SWCNT assembly was observed after the deposition process. On the other hand, all SC and SDeC cases show dense SWCNT assemblies. In the SC surfactant case, similar dense assemblies of SWCNTs is observed even with phosphate buffered saline (PBS, $2 \times$ ) and 4-(2-hydroxyethyl)-1-piperazineethanesulfonic acid (HEPES, $40 \mathrm{mM}$ ) buffers. The assembled SWCNTs from SC solutions are identified via Raman spectroscopy as well, where a clear $G$ 


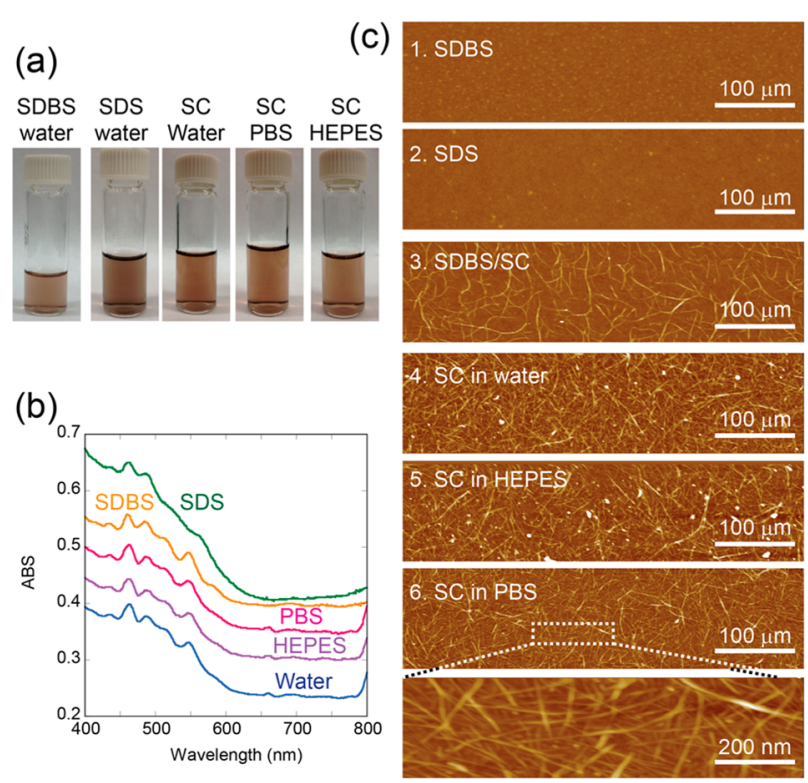

Figure 2. (a) Pictures of SWCNT suspensions from different surfactant solutions. Solutions with SDBS and SDS surfactants are suspended in Milli-Q water, while the SC surfactants are in Milli-Q water, PBS, and HEPES buffers. (b) Optical spectra of SWCNT suspensions in a range of $400-800 \mathrm{~nm}$. Peaks around $450 \mathrm{~nm}$ are van Hove transitions which indicate isolated SWCNTs in the suspensions. (c) AFM images after the deposition of the suspensions for $10 \mathrm{~min}$ at room temperature. Images are from SDBS solution (1), SDS solution (2), a mixed solution of SDBS and SC (1:1 mol:mol, 3), SC solution in Milli-Q water (4), SC solution in $40 \mathrm{mM}$ HEPES (5), and SC suspension in $2 \times$ PBS (6). While SDBS and SDS prohibit nanotube assembly, highly dense films are obtained with SC as the surfactant.

band peak at $1600 \mathrm{~cm}^{-1}$ is observed with a small $D$ band peak at $1350 \mathrm{~cm}^{-1}$ (Figure S2). ${ }^{66}$ Even though the SDBS surfactant prohibits assembly, the addition of SC surfactant into the SDBS solution enables the assembly of SWCNTs (Figures 2c and 3). According to the above results, AS prohibit the assembly of SWCNTs, while SS clearly facilitate the assembly process. These results are examined on PLL modified surfaces, and the trend is similar on (3-aminopropyl)triethoxysilane (APTES) modified surfaces as well (Figure S3).

Controlled experiments are performed to analyze the differences in the assembly processes between AS and SS. Assembled structures of these surfactants onto $\mathrm{Si} / \mathrm{SiO}_{2}$ substrates in the absence of nanotubes are examined. To visualize the surfactant layer formation on the $\mathrm{SiO}_{2}$ surface, a fluorescent lipid dye, octadecyl rhodamine B chloride (R18, 1 $\mathrm{mg} / \mathrm{mL}$ in ethanol), was used. ${ }^{67}$ The dye was mixed with the surfactant solutions or Milli-Q water with a volume ratio of surfactant solution:R18 solution of 50:1 followed by deposition of the solution onto a PLL modified $\mathrm{SiO}_{2} / \mathrm{Si}$ wafer. As observed in Figures 3a and S4, a corresponding fluorescence from R18 on the substrate can only be observed for SDBS and SDS surfactant solutions. This result indicates that both SDBS and SDS form a layered assembly on the substrate surface incorporating R18. ${ }^{68}$ Since the SWCNT surface is also covered with these lipids, the electrostatic repulsion force causes the prohibition of SWCNT assembly when using SDS or SDBS as the surfactants (Figure 3d), consistent with the observed assembly results of Figure 2 .

To further investigate the SWCNT adhesion mechanism, we used a dye perylene which has a similar aromatic surface like
SWCNTs. Fluorescence microscope images were obtained after deposition of mixtures of the surfactant solutions and a saturated solution of perylene in ethanol followed by washing with Milli-Q water and $\mathrm{N}_{2}$ gas drying. Only the SS case shows obvious fluorescent from perylene (Figures $3 \mathrm{~b}$ and S4). Since extremely small fluorescence was observed from the perylene dispersed in water (PLL/-), interactions between the aromatic moiety and PLL modified surface can be concluded as very weak. This result indicates that only through SS can perylene exhibit adequate adhesion allowing subsequent attachment onto the substrate surface (Figure 3e). For further analysis of the effective adhesion of SWCNTs shown in SS, a dye with a similar steroid moiety, 23-(dipyrrometheneboron difluoride)24-norcholesterol, was examined (Figure 3c). The surfactant solutions, or Milli-Q water, were mixed with an ethanol solution $(1 \mathrm{mg} / \mathrm{mL})$ of the steroid-dye and cast onto the PLL modified substrate. After the deposition, the substrate was washed with Milli-Q water and dried in air. As shown in Figure $3 c$, only water (no surfactant, PLL/-) and SS (PLL/SC) show a corresponding fluorescent signal. These results indicate that (i) the steroid moiety can attach onto the PLL surface via effective van der Waals interactions, (ii) SS does not prohibit the absorption of the steroid dye, and (iii) no adhesion of the dye is observed in AS solutions since the steroid-dye is wrapped with AS as shown in the perylene cases. In summary, we can conclude the following points: SS can attach onto the modified surfaces, and SS does not prevent the absorption or adhesion of other SS and SS encapsulated SWCNTs (and perylene) onto the amine-functionalized substrate surface (Figure $3 \mathrm{e}$ ). In addition, SS are able to isolate/disperse SWCNTs very well as reported previously; ${ }^{58}$ our solutions also show similar stability of SWCNT suspension for at least more than 2 weeks (Figure S5). These results indicate the stability of SWCNT solutions without bundling through SS. Thus, SS is ideal for assembling SWCNT dense networks.

Capacitance-voltage $(C-V)$ measurements were performed to quantify the assembled SWCNT density when using SS as the surfactant, with the setup illustrated in Figure 4a. Source (S) and drain (D) electrodes are bridged by the percolated SWCNT network on $50 \mathrm{~nm}$ of $\mathrm{SiO}_{2}$ on a heavily doped $p+-\mathrm{Si}$ wafer, which acts as a universal gate electrode. The S/D electrodes are electrically grounded, and a voltage is applied to the back gate while measuring the gate capacitance. The measured capacitance consists of the gate oxide capacitance and the parasitic capacitance arising from the gate-S/D overlap. To remove the parasitic capacitance component, devices with four channel lengths $(L)$ from 10 to $100 \mu \mathrm{m}$ at a fixed width $(W)$ of $200 \mu \mathrm{m}$ were fabricated and measured (Figure $4 \mathrm{~b}$ ). Figure $4 \mathrm{c}$ shows the linear correlation of the total capacitance vs $L$ for a deposition time of $70 \mathrm{~min}$ at room temperature (data for other deposition times are shown in Figures S6 and S7). The gate-S/ $\mathrm{D}$ overlap capacitance is the $y$-intercept of this plot. Thus, the gate oxide capacitance under the SWCNT network, $C_{\text {channel }}$, can be extracted. From the capacitance measurements, the effective areal coverage of the SWCNTs can be defined by the following equation:

$$
\text { effective coverage }=C_{\text {channel }} /\left(\varepsilon_{0} \varepsilon_{\text {ox }} / t_{\text {ox }}\right)
$$

where $\varepsilon_{0} \varepsilon_{\mathrm{ox}}=3.9 \times 8.85^{-14} \mathrm{~F} / \mathrm{cm}$ and $t_{\mathrm{ox}}=50 \mathrm{~nm}$ is used for the dielectric constant and thickness of the $\mathrm{SiO}_{2}$, respectively. Here, an effective coverage of $100 \%$ would correspond to a nanotube network that would yield a $C_{\text {channel }}$ that is defined by the parallel plate capacitance of the gate oxide $\left(\varepsilon_{0} \varepsilon_{\mathrm{ox}} / t_{\mathrm{ox}}\right)$. In 
(a) Lipid dye
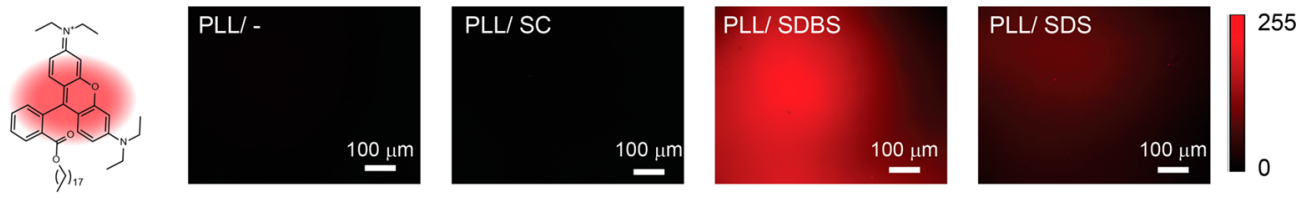

(b) Aromatic dye
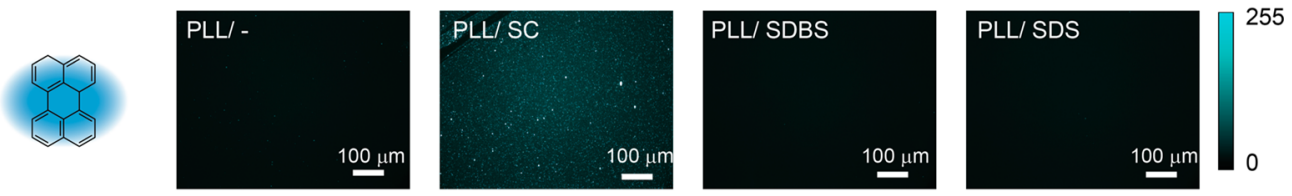

(c)
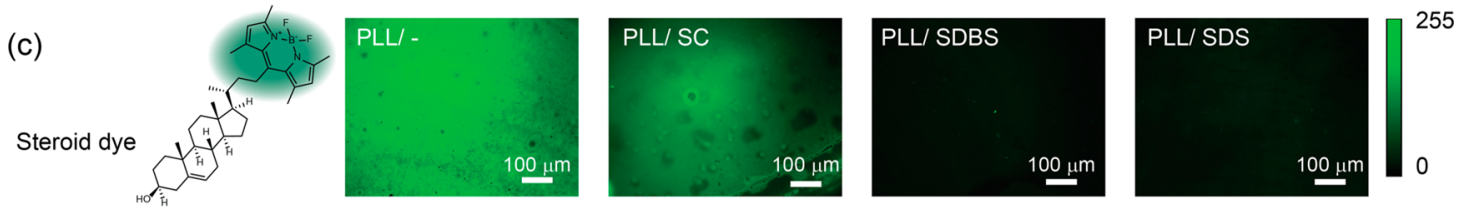

(d)
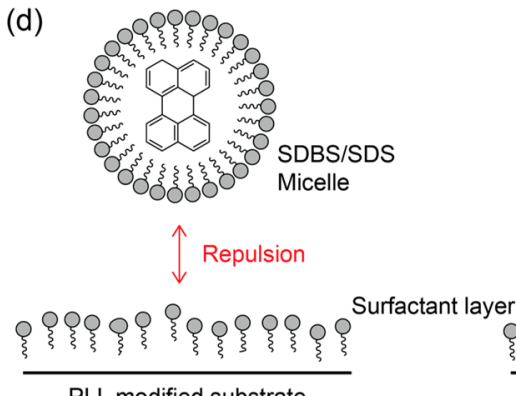

PLL modified substrate
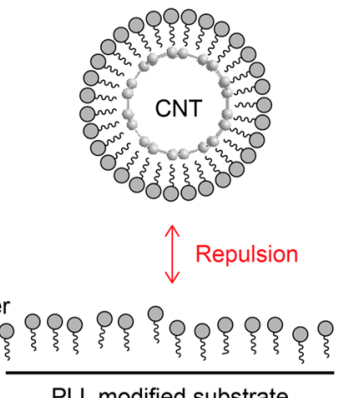

(e)

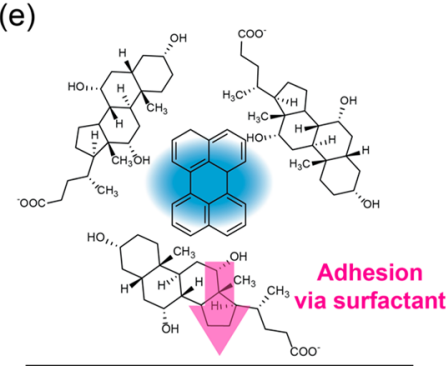

PLL modified substrate

Figure 3. (a-c) Fluorescent microscope images using (a) lipid dye (R18), (b) aromatic dye (perylene) and (c) steroid-dye (23(dipyrrometheneboron difluoride)-24-norcholesterol). Images are obtained from the deposition of a mixed solution of each surfactant and dye onto a PLL modified $\mathrm{SiO}_{2} / \mathrm{Si}$ wafer. PLL/-, PLL/SC, PLL/SDBS, and PLL/SDS represent the process with no surfactant (Milli-Q water), SC, SDBS, and SDS surfactants, respectively. Colors are pseudocolor, and the intensity range is between 0 to 255 . (d) Schematic illustration of the repulsive force between aromatic molecules wrapped with AS (left: perylene and right: SWCNT) and an AS assembly on the surface of the substrate. (e) Mechanism of the adhesion of the aromatic molecule, perylene (model molecule of SWCNTs), onto the surface of the substrate. Perylene sticks on the surface through the SS surfactant.

other words, at $100 \%$ effective coverage, the nanotube network resembles a continuous conducting film, at least from a capacitance point of view. This presents a simple approach to quantify the nanotube density, without having to rely on imaging techniques to analyze density which can be arbitrary. Figure $4 \mathrm{~d}$ depicts the deposition time dependence of the extracted $C_{\text {channel }}$ and the effective coverage area of SWCNTs estimated from eq 1 . The effective coverage reaches $80 \%$ with deposition times as short as $5 \mathrm{~min}$ and monotonically increases to $\sim 90 \%$ as the deposition time is increased to $70 \mathrm{~min}$.

Transfer characteristic curves of TFTs obtained from the nanotube networks of Figure $4 \mathrm{~d}$ are shown in Figure S8. The ON current level is increased from $10^{-7}$ to $10^{-6} \mathrm{~A} \mu \mathrm{m}^{-1}$ at $L=$ $10 \mu \mathrm{m}$ as the deposition time is increased from 1 to $70 \mathrm{~min}$. For deposition times between 1 and $10 \mathrm{~min}$, ON/OFF ratios of up to $10^{5}$ can be obtained. In terms of uniformity of the OFF current levels, deposition times between 1 and 10 min show higher uniformity than that of $70 \mathrm{~min}$. This is due to the higher amount of nanotube bundling and metallic SWCNTs that are deposited in the 70 min case which increases the OFF current and decreases the uniformity of the transfer characteristics of the TFTs. ${ }^{39}$ Different nanotube solutions of Milli-Q, PBS and HEPES exhibit similar device performance (Figure S9).

To estimate the number of SWCNTs assembled onto the surface in a unit area (number of SWCNTs/ $\mu \mathrm{m}$ ), we used the following analytical equation: ${ }^{61,69}$

$$
C_{\text {channel }}=\left\{C_{\mathrm{Q}}^{-1}+\frac{1}{2 \pi \varepsilon_{0} \varepsilon_{o x}} \ln \left[\frac{\Lambda_{0}}{R} \frac{\sinh \left(\frac{2 \pi t_{\mathrm{ox}}}{\Lambda_{0}}\right)}{\pi}\right]\right\}^{-1} \Lambda_{0}^{-1}
$$

where $1 / \Lambda_{0}$ represents the density of SWCNTs (number $/ \mu \mathrm{m}$ ) and $C_{\mathrm{Q}}=4.0 \times 10^{-10} \mathrm{~F} / \mathrm{m}$ is the quantum capacitance of SWCNTs. For the radius of the SWCNTs, $R=0.7 \mathrm{~nm}$ was used. The calculated curve of $C_{\text {channel }}$ vs SWCNT density is plotted in Figure 4e. From the plot, the estimated number of SWCNTs is obtained for various deposition times. The density of SWCNTs varies from 5 to $\sim 60 \mu \mathrm{m}^{-1}$ for deposition times from 1 to $70 \mathrm{~min}$. The density of $60 \mu \mathrm{m}^{-1}$ corresponds to an arrangement of SWCNTs at every $16 \mathrm{~nm}$. This result indicates high-density arrangement of SWCNTs that can be readily obtained via PLL modification of the substrate and SS dispersion of nanotubes.

While the obtained density of SWCNTs is quite high using the SS process discussed above, the required deposition time is relatively long, which limits the potential throughput of the process. To shorten the assembly time for SWCNTs, we examined the effect of deposition temperature. As the deposition temperature is increased to $70{ }^{\circ} \mathrm{C}$, suspensions of SC show a denser SWCNT assembly and the effective coverage increases up to $99 \%$ for a deposition time of only $10 \mathrm{~min}$ 
(a)
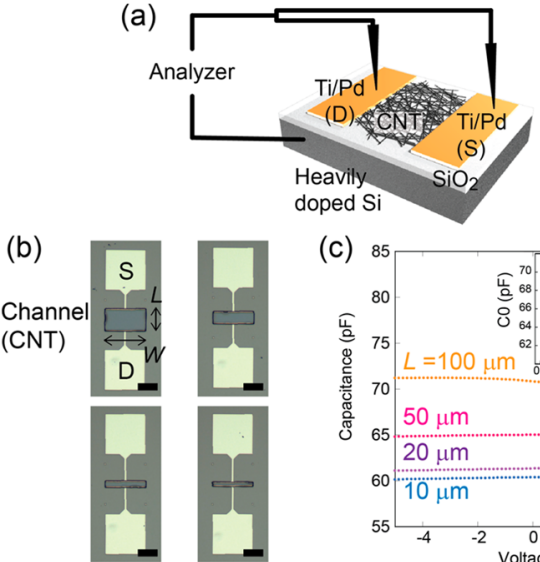

(d)

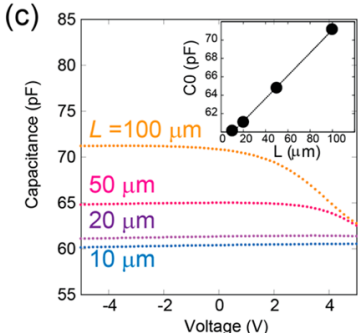

(e)
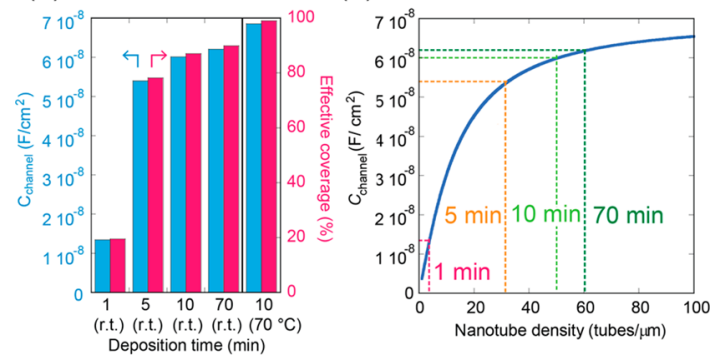

Figure 4. Quantification of the SWCNT density. (a) Schematic illustration of the setup for measuring $C-V$ of SWCNT TFTs. The device structure consists of S/D electrodes bridged by the SWCNT network on top of thermally grown $\mathrm{SiO}_{2}$ on heavily doped $\mathrm{Si}$ as the back gate. The capacitance is measured between the $\mathrm{Si}$ substrate and the S/D electrodes. (b) Microscope images of the devices with various channel lengths $(L)$. Devices have $L=10,20,50$, and $100 \mu \mathrm{m}$ and width $(W)=200 \mu \mathrm{m}$. Scale bars are $100 \mu \mathrm{m}$. (c) $C-V$ curves with $L=$ $10,20,50$, and $100 \mu \mathrm{m}$ for the devices from SWCNT suspensions in SC deposited for $70 \mathrm{~min}$ at room temperature. Inset is a plot of capacitance at $-5 \mathrm{~V}(\mathrm{CO})$ vs channel length $(\mathrm{L})$. (d) Calculated capacitance of the CNT network channel $\left(C_{\text {channel }}\right)$ and effective surface coverage obtained from $C-V$ measurements for 1 to $70 \mathrm{~min}$ deposition at room temperature (rt) and $10 \mathrm{~min}$ deposition at $70{ }^{\circ} \mathrm{C}$. (e) Capacitance $\left(C_{\text {channel }}\right)$ vs SWCNT density (tubes/ $\left.\mu \mathrm{m}\right)$ plot estimated from eq 2 and the corresponding tube density for each deposition time. Tube density varies from 5 to 60 (tubes/ $\mu \mathrm{m}$ ).

(Figure 4d). This value corresponds to over 230 nanotubes $/ \mu \mathrm{m}$ (Figure S10e). Shorter assembly time is observed at higher temperature due to an increased rate of surfactants exchange at the surface that facilitates the probability for adhesion of SS encapsulated SWCNTs. Even in a suspension of SDBS, for which no SWCNT assembly could be observed at room temperature, a higher temperature of $70{ }^{\circ} \mathrm{C}$ induces deposition of SWCNTs (Figure S10), though the SWCNT density is low. This again suggests the effect of temperature in enhancing the SWCNT assembling rate at the surface of the substrate.

When our high-temperature assembly process is combined with the optimized SS surfactant, a feasible R2R assembly process can be enabled to fully substantiate the vast potential of our proposed techniques. (Figure 5a and Movie 1). A cartridge of polyethylene telephthalate (PET) film is fed through (i) PLL solution, (ii) Milli-Q water to wash any extra PLL, (iii) SWCNT suspension on a hot plate $\left(70^{\circ} \mathrm{C}\right)$, and (iv) a final Milli-Q water rinse with a feeding speed about $10 \mathrm{~cm} / \mathrm{min}$. A drying fan is placed after the first Milli- $Q$ bath to dry samples prior to the SWCNT bath in order to avoid dilution of the SWCNT solution. Figure $5 \mathrm{~b}$ shows a $1 \mathrm{~m}$ long PET film with

assembled SWCNTs on the surface via the R2R process. It should be noted that the speed of our R2R process is not limited by the deposition rate of the SWCNTs, but rather the volume of SWCNT solution used. If the volume of SWCNTs (i.e., the bath length) is increased as would be necessary for a commercial scale, the throughput of the process could be linearly increased. AFM images indicate that SWCNTs are successfully deposited onto the flexible PET substrate (Figure 5c). The transfer characteristic of a device obtained from this process is shown in Figure 5d. The device is a top gate structure with $40 \mathrm{~nm}$ palladium $\mathrm{S} / \mathrm{D}$ electrodes and $40 \mathrm{~nm}$ gold gate electrodes. The channel width and length are $W=2.5 \mathrm{~mm}$ and $L=125 \mu \mathrm{m}$, respectively. Parylene ( $700 \mathrm{~nm}$ in thickness) was used as the gate dielectric layer. The peak mobility from a parallel plate model is extracted as $2 \mathrm{~cm}^{2} / \mathrm{V} \mathrm{s}$ (Supporting Information), which is respectable for a R2R process scheme, but lower than those obtained by solution casting on smooth surfaces. ${ }^{39,61}$ We speculate that the roughness of the PET film reduces the density of the assembled SWCNTs. For higher device performance, contact metals, deposition process, dielectric layer, etc., need to be further optimized. Previously, we demonstrated a scalable process for fabricating SWCNTbased TFT arrays via gravure printing. ${ }^{36}$ However, the SWCNT deposition process using commercially available solutions required several hours of solution casting in order to obtain a high density. The work here provides the ability to control and drastically lower the deposition time for nanotube networks, making it more viable for R2R processing of SWCNT TFTs.

\section{CONCLUSION}

We have demonstrated a highly dense and fast solution-based SWCNT assembly viable for scalable TFT fabrication. The key finding is that steroid-based surfactants facilitate dense assembly of SWCNTs on amine functionalized surfaces, while alkyl surfactants prohibit nanotube assembly. The difference is attributed to the packing nature of these two types of surfactants on the substrate surface, given the existence of large quantities of residual surfactants in SWCNT suspensions. Furthermore, deposition density and rate are shown to increase with temperature as it affects surfactant-substrate interactions. By using this knowledge, fast SWCNT assembly with an effective surface coverage of up to $99 \%$ is obtained as characterized by $C-V$ measurements. The utility of this process is demonstrated by a R2R assembly of SWCNTs on large-area PET substrates, with the nanotube films used to fabricate TFTs. This investigation opens new routes toward the realization of large-area, flexible electronics using SWCNT-based thin films.

\section{EXPERIMENTAL SECTION}

Making the SWCNT Suspension. SWCNTs are obtained from Nanointegris Inc. A $0.04 \mathrm{mg}$ entangled sheet of SWCNTs (99\% semiconducting) is immersed into $4 \mathrm{~mL}$ surfactant solutions ( 30 $\mu \mathrm{mol} / \mathrm{mL}$ ). All surfactants are from Sigma-aldrich. PBS and HEPES are from Gibco, both of which are diluted with Milli-Q water to make PBS $(2 \times)$ and HEPES $(40 \mathrm{mM})$ before use. Bath sonication is applied for $30 \mathrm{~min}$ to break up the sheet into tiny pieces followed by sonication with a probe sonicator (Cole-Parmer) at $150 \mathrm{~W}$ to isolate the nanotubes. The program of the probe sonication is set to alternately turn on $(1 \mathrm{~s})$ and off $(1 \mathrm{~s})$ for a total of $80 \mathrm{~min}$. The bath temperature is kept below $10{ }^{\circ} \mathrm{C}$ using cold water to prevent evaporation of the solution. After probe sonication, the solution was placed into a bath sonicator for another $30 \mathrm{~min}$ and then kept at room temperature for at least $12 \mathrm{~h}$. Any precipitates were decanted, and the supernatant SWCNT suspension was used for deposition. 


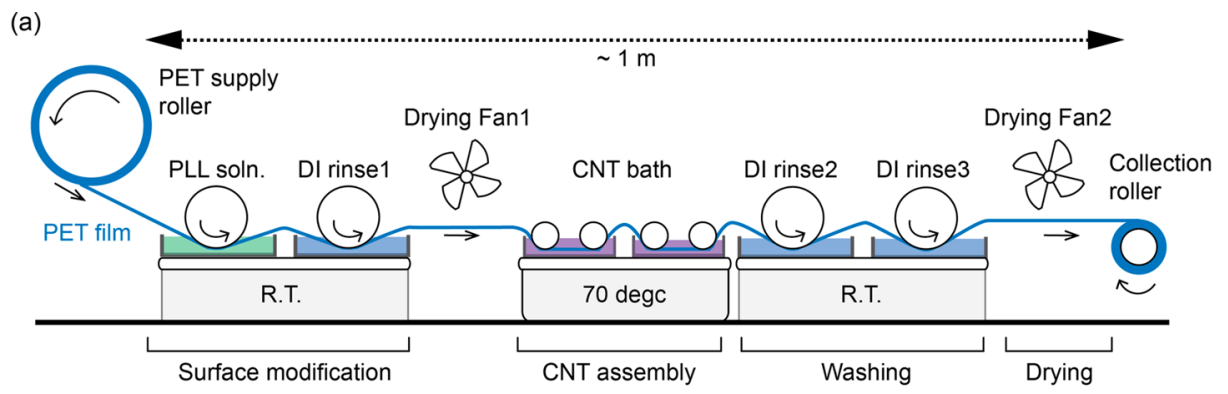

(b)

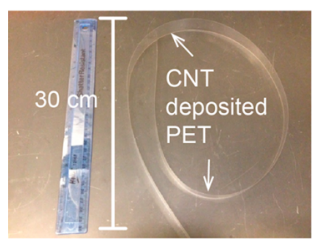

(c)

$\sim 10-20 \mathrm{~cm}$ from the top

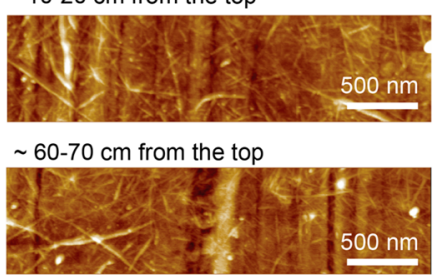

(d)

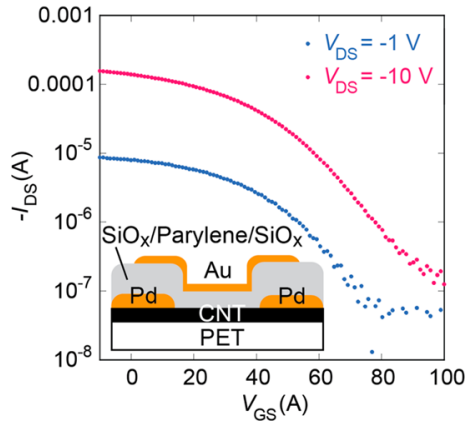

Figure 5. (a) Schematic illustration of the R2R SWCNT assembly process. The setup is constructed from PET supply and collection rollers with baths of PLL solution, DI rinse 1, SWCNT deposition, and DI rinses 2 and 3. DI rinse 1 is for washing away extra PLL, DI rinses 2 and 3 are for washing excess SWCNT solution. Drying fans 1 and 2 are placed before the SWCNT baths and after the final DI rinse bath, respectively. (b) Macroscopic picture of a SWCNT deposited PET film. The length is about $1 \mathrm{~m}$. (c) AFM images of deposited SWCNTs on the PET film around 10-20 cm (top image) and 60-70 cm (bottom image) from the beginning of deposition. (d) Transfer characteristic curves obtained from a transistor made with R2R assembled SWCNT networks with $W=2.5 \mathrm{~mm}$ and $L=125 \mu \mathrm{m}$.

Fabrication of the Devices. Devices (Figures 3, S8 and S9) are fabricated with standard lithographic techniques using $\mathrm{S} 1818$ photoresist. $\mathrm{A} \mathrm{SiO}_{2} /$ heavily doped $p^{+}$-Si wafer was treated $\mathrm{O}_{2}$ plasma $(120$ $\mathrm{W}, 2 \mathrm{~min})$, and then PLL solution (0.1\%, Sigma-Aldrich) was deposited for $5 \mathrm{~min}$ followed by rinse with Milli-Q water. SWCNT suspension was cast onto the PLL modified wafer followed by DI rinse. The deposited wafers were annealed in vacuum at $200{ }^{\circ} \mathrm{C}$ for $2 \mathrm{~h}$. $\mathrm{Pd}$ source, drain, and Au gate metals were all deposited via electron beam evaporation followed by etching extra SWCNTs with $\mathrm{O}_{2}$ plasma so that the SWCNT network only remains between the source and drain electrodes. The device shown in Figure $5 \mathrm{~d}$ is made using a shadow mask $(W=2.5 \mathrm{~mm}, L=125 \mu \mathrm{m})$ with $\mathrm{Pd}(40 \mathrm{~nm})$ source/drain contacts followed by $\mathrm{SiO}_{x} /$ parylene $\mathrm{C} / \mathrm{SiO}_{x}(10 / \sim 700 / 10 \mathrm{~nm})$ deposition as a gate dielectric; the $\mathrm{SiO}_{x}$ was deposited via electron beam evaporation and the parylene $\mathrm{C}$ was deposited via a CVD method. The top gate metal $(\mathrm{Au}, 40 \mathrm{~nm})$ is deposited via electron beam evaporation. The device was annealed to improve source and drain metal contacts at $160^{\circ} \mathrm{C}$ for $1 \mathrm{~h}$ on a hot plate.

Characterization. Fluorescent microscope images are taken using an Axioimager (Zeiss) equipped with a Hamamatsu Orca 03 CCD camera. The images are analyzed with ImageJ software. The dyes for visualization were purchased from Sigma-Aldrich (Perylene), Biotium Inc. (R18), and Avanti Polar Lipids Inc. (23-(dipyrrometheneboron difluoride)-24-norcholesterol). Microscope images were taken using an Olympus BX51 microscope equipped with a digital camera (Olympus, QCOLOR3). Characteristic curves were obtained with an HP 4155C analyzer with a probe station. The capacitance-voltage measurements were obtained from an Agilent B1500a analyzer with a probe station. Raman spectroscopy was conducted with a Thermo-Scientific DXR.

\section{ASSOCIATED CONTENT}

\section{S Supporting Information}

Details for calculation of the field-effect mobility, R2R setup, AFM images from CNT solutions, Raman spectra for deposited CNTs, optical spectra of CNT suspension with SC surfactant, $C-V$ data for calculating density of CNTs, and drain current vs gate voltage curves. This material is available free of charge via the Internet at http://pubs.acs.org.

\section{AUTHOR INFORMATION}

\section{Corresponding Author}

ajavey@berkeley.edu

\section{Present Address}

"Department of Electronic Materials Engineering, Kwangwoon University, Seoul 139-701, Republic of Korea

\section{Notes}

The authors declare no competing financial interest.

\section{ACKNOWLEDGMENTS}

This work was supported by NSF NASCENT Center.

\section{REFERENCES}

(1) Dresselhaus, M. S.; Dresselhaus, G.; Saito, R. Carbon 1995, 33, 883.

(2) Carbon Nanotube Electronics; Javey, A., Kong, J., Eds.; Springer: New York, 2009.

(3) Niyogi, S.; Hamon, M. A.; Hu, H.; Zhao, B.; Bhowmik, P.; Sen, R.; Itkis, M. E.; Haddon, R. C. Acc. Chem. Res. 2002, 35, 1105.

(4) Durkop, T.; Getty, S. A.; Cobas, E.; Fuhrer, M. S. Nano Lett. 2004, 4, 35 .

(5) Davis, J. J.; Coleman, K. S.; Azamian, B. R.; Bagshaw, C. B.; Green, M. L. H. Chem.-Eur. J. 2003, 9, 3732.

(6) Baughman, R. H.; Zakhidov, A. A.; de Heer, W. A. Science 2002, 297, 787.

(7) Yu, M. F.; Files, B. S.; Arepalli, S.; Ruoff, R. S. Phys. Rev. Lett. 2000, 84, 5552 .

(8) Moon, H. K.; Lee, S. H.; Choi, H. C. ACS Nano 2009, 3, 3707.

(9) Zhang, X.; Yu, Z. B.; Wang, C.; Zarrouk, D.; Seo, J. W. T.; Cheng, J. C.; Buchan, A. D.; Takei, K.; Zhao, Y.; Ager, J. W.; Zhang, J. J.; Hettick, M.; Hersam, M. C.; Pisano, A. P.; Fearing, R. S.; Javey, A. Nat. Commun. 2014, 5, 2983. 
(10) Rueckes, T.; Kim, K.; Joselevich, E.; Tseng, G. Y.; Cheung, C. L.; Lieber, C. M. Science 2000, 289, 94.

(11) Cao, Q.; Rogers, J. A. Adv. Mater. 2009, 21, 29.

(12) Kim, S. N.; Rusling, J. F.; Papadimitrakopoulos, F. Adv. Mater. 2007, 19, 3214.

(13) Rutherglen, C.; Jain, D.; Burke, P. Nat. Nanotechnol. 2009, 4, 811.

(14) Misewich, J. A.; Martel, R.; Avouris, P.; Tsang, J. C.; Heinze, S.; Tersoff, J. Science 2003, 300, 783.

(15) Tenent, R. C.; Barnes, T. M.; Bergeson, J. D.; Ferguson, A. J.; To, B.; Gedvilas, L. M.; Heben, M. J.; Blackburn, J. L. Adv. Mater. 2009, 21, 3210.

(16) Wang, F.; Gu, H. W.; Swager, T. M. J. Am. Chem. Soc. 2008, 130, 5392

(17) Li, J.; Lu, Y.; Ye, Q.; Cinke, M.; Han, J.; Meyyappan, M. Nano Lett. 2003, 3, 929.

(18) Kong, J.; Franklin, N. R.; Zhou, C.; Chapline, M. G.; Peng, S.; Cho, K. J.; Dai, H. J. Science 2000, 287, 622.

(19) Avouris, P.; Chen, Z.; Perebeinos, V. Nat. Nanotechnol. 2007, 2, 605.

(20) Kaempgen, M.; Chan, C. K.; Ma, J.; Cui, Y.; Gruner, G. Nano Lett. 2009, 9, 1872.

(21) Wang, C.; Takei, K.; Takahashi, T.; Javey, A. Chem. Soc. Rev. 2013, 42, 2592.

(22) Sun, D. M.; Timmermans, M. Y.; Tian, Y.; Nasibulin, A. G.; Kauppinen, E. I.; Kishimoto, S.; Mizutani, T.; Ohno, Y. Nat. Nanotechnol. 2011, 6, 156.

(23) Flavel, B. S.; Kappes, M. M.; Krupke, R.; Hennrich, F. ACS Nano 2013, 7, 3557.

(24) Ju, S. Y.; Doll, J.; Sharma, I.; Papadimitrakopoulos, F. Nat. Nanotechnol. 2008, 3, 356.

(25) Arnold, M. S.; Green, A. A.; Hulvat, J. F.; Stupp, S. I.; Hersam, M. C. Nat. Nanotechnol. 2006, 1, 60.

(26) Zhang, D. H.; Ryu, K.; Liu, X.; Polikarpov, E.; Ly, J.; Tompson, M. E.; Zhou, C. Nano Lett. 2006, 6, 1880.

(27) Meitl, M. A.; Zhou, Y. X.; Gaur, A.; Jeon, S.; Usrey, M. L.; Strano, M. S.; Rogers, J. A. Nano Lett. 2004, 4, 1643.

(28) Park, S.; Vosguerichian, M.; Bao, Z. A. Nanoscale 2013, 5, 1727.

(29) Rouhi, N.; Jain, D.; Zand, K.; Burke, P. J. Adv. Mater. 2011, 23, 94.

(30) Sangwan, V. K.; Ortiz, R. P.; Alaboson, J. M. P.; Emery, J. D.; Bedzyk, M. J.; Lauhon, L. J.; Marks, T. J.; Hersam, M. C. ACS Nano 2012, 6, 7480

(31) LeMieux, M. C.; Roberts, M.; Barman, S.; Jin, Y. W.; Kim, J. M.; Bao, Z. N. Science 2008, 321, 101.

(32) Park, H.; Afzali, A.; Han, S. J.; Tulevski, G. S.; Franklin, A. D.; Tersoff, J.; Hannon, J. B.; Haensch, W. Nat. Nanotechnol. 2012, 7, 787.

(33) Wang, C.; Zhang, J.; Ryu, K. M.; Badmaev, A.; De Arco, L. G.; Zhou, C. W. Nano Lett. 2009, 9, 4285.

(34) Wang, C.; Hwang, D.; Yu, Z. B.; Takei, K.; Park, J.; Chen, T.; Ma, B. W.; Javey, A. Nat. Mater. 2013, 12, 899.

(35) Jung, M.; Kim, J.; Noh, J.; Lim, N.; Lim, C.; Lee, G.; Kim, J.; Kang, H.; Jung, K.; Leonard, A. D.; Tour, J. M.; Cho, G. IEEE Trans. Electron Devices 2010, 57, 571.

(36) Lau, P. H.; Takei, K.; Wang, C.; Ju, Y.; Kim, J.; Yu, Z. B.; Takahashi, T.; Cho, G.; Javey, A. Nano Lett. 2013, 13, 3864.

(37) Kim, B.; Jang, S.; Prabhumirashi, P. L.; Geier, M. L.; Hersam, M. C.; Dodabalapur, A. Appl. Phys. Lett. 2013, 103, 082119.

(38) Kumar, S.; Murthy, J. Y.; Alam, M. A. Phys. Rev. Lett. 2005, 95, 066802.

(39) Takahashi, T.; Takei, K.; Gillies, A. G.; Fearing, R. S.; Javey, A. Nano Lett. 2011, 11, 5408.

(40) Engel, M.; Small, J. P.; Steiner, M.; Freitag, M.; Green, A. A.; Hersam, M. C.; Avouris, P. ACS Nano 2008, 2, 2445.

(41) Wang, Y. B.; Iqbal, Z.; Mitra, S. J. Am. Chem. Soc. 2006, 128, 95.

(42) Pénicaud, A.; Poulin, P.; Derré, A.; Anglaret, E.; Petit, P. J. Am. Chem. Soc. 2005, 127, 8.

(43) Hecht, D. S.; Heintz, A. M.; Lee, R.; Hu, L.; Moore, B.; Cucksey, C.; Risser, S. Nanotechnology 2011, 22, 075201.
(44) Davis, V. A.; Parra-Vasquez, A. N. G.; Green, M. J.; Rai, P. K.; Behabtu, N.; Prieto, V.; Booker, R. D.; Schmidt, J.; Kesselman, E.; Zhou, W.; Fan, H.; Adams, W. W.; Hauge, R. H.; Fischer, J. E.; Cohen, Y.; Talmon, Y.; Smalley, R. E.; Pasquali, M. Nat. Nanotechnol. 2009, 4, 830.

(45) Vaisman, L.; Wagner, H. D.; Marom, G. Adv. Col. Int. Sci. 2006, $128,37$.

(46) O'Connell, M. J.; Boul, P.; Ericson, L. M.; Huffman, C.; Wang, Y.; Haroz, E.; Kuper, C.; Tour, J.; Ausman, K. D.; Smalley, R. E. Chem. Phys. Lett. 2001, 342, 265.

(47) Wang, J.; Musameh, M.; Lin, Y. J. Am. Chem. Soc. 2003, 125, 2408.

(48) Murakami, H.; Nomura, T.; Nakashima, N. Chem. Phys. Lett. 2003, 378, 481.

(49) Wang, D.; Chen, L. Nano Lett. 2007, 7, 1480.

(50) Yan, L. Y.; Poon, Y. F.; Chan-Park, M. B.; Chen, Y.; Zhang, Q. J. Phys. Chem. C 2008, 112, 7579.

(51) Lin, D.; Xing, B. Environ. Sci. Technol. 2008, 42, 5917.

(52) Islam, M. F.; Rojas, E.; Bergey, D. M.; Johnson, A. T.; Yodh, A. G. Nano Lett. 2003, 3, 269.

(53) Jiang, L.; Gao, L.; Sun, J. J. Colloid Interface Sci. 2003, 260, 89.

(54) Shin, J. Y.; Premkumar, T.; Geckeler, K. E. Chem.-Eur. J. 2008, 14, 6044 .

(55) Paredes, J. I.; Burghard, M. Langmuir 2004, 20, 5149.

(56) Nish, A.; Hwang, J. Y.; Doig, J.; Nicholas, R. J. Nat. Nanotechnol. 2007, 2, 640 .

(57) Matarredona, O.; Rhoads, H.; Li, Z.; Harwell, J. H.; Balzano, L.; Resasco, D. E. J. Phys. Chem. B 2003, 107, 13357.

(58) Wenseleers, W.; Vlasov, I. I.; Goovaerts, E.; Obraztsova, E. D.; Lobach, A. S.; Bouwen, A. Adv. Funct. Mater. 2004, 14, 1105.

(59) Moore, V. C.; Strano, M. S.; Haroz, E. H.; Hauge, R. H.; Smalley, R. E.; Schmidt, J.; Talmon, Y. Nano Lett. 2003, 3, 1379.

(60) Moshammer, K.; Hennrich, F.; Kappes, M. M. Nano Res. 2009, 2, 599 .

(61) Wang, C.; Chien, J. C.; Takei, K.; Takahashi, T.; Nah, J.; Niknejad, A. M.; Javey, A. Nano Lett. 2012, 12, 1527.

(62) Taffarel, S. R.; Rubio, J. Miner. Eng. 2010, 23, 771.

(63) Shen, D. Z.; Kang, Q.; Zhang, X. L.; Li, W. P.; Liu, Z. C. Mikrochim. Acta 2002, 138, 89.

(64) Verde, A. V.; Frenkel, D. Soft Matter 2010, 6, 3815.

(65) O'Connell, M. J.; Bachilo, S. M.; Huffman, C. B.; Moore, V. C.; Strano, M. S.; Haroz, E. H.; Rialon, K. L.; Boul, P. J.; Noon, W. H.; Kittrell, C.; Ma, J.; Hauge, R. H.; Weisman, R. B.; Smalley, R. E. Science 2002, 297, 593.

(66) Dresselhaus, M. S.; Dresselhaus, G.; Saito, R.; Jorio, A. Phys. Rep. 2005, 409, 47.

(67) Kiriya, D.; Ikeda, M.; Onoe, H.; Takinoue, M.; Komatsu, H.; Shimoyama, Y.; Hamachi, I.; Takeuchi, S. Angew. Chem., Int. Ed. 2012, $51,1553$.

(68) Deng, Y.; Wang, Y.; Holtz, B.; Li, J.; Traaseth, N.; Veglia, G.; Stottrup, B. J.; Elde, R.; Pei, D. Q.; Guo, A.; Zhu, X. Y. J. Am. Chem. Soc. 2008, 130, 6267.

(69) Cao, Q.; Xia, M.; Kocabas, C.; Shim, M.; Rogers, J. A.; Rotkin, S. V. Appl. Phys. Lett. 2007, 90, 023516. 


\section{Supporting Information}

\section{Design of surfactant-substrate interactions for roll-to-roll assembly of carbon nanotubes for thin film transistors}

Daisuke Kiriya $^{1,23}$, Kevin Chen ${ }^{1,2,3}$, Hiroki Ota ${ }^{1,2,3}$, Yongjing Lin ${ }^{1,2,3}$, Peida Zhao ${ }^{1,2,3}$, Zhibin Yu ${ }^{1,2,3}$, Tae-jun $\mathrm{Ha}^{1,2,3}$ and Ali Javey ${ }^{1,2,3^{*}}$

${ }^{1}$ Electrical Engineering and Computer Sciences, and Berkeley Sensor and Actuator Center, University of California at Berkeley, Berkeley, California 94720, United States

${ }^{2}$ Materials Sciences Division, Lawrence Berkeley National Laboratory, Berkeley, California 94720, United States

${ }^{3}$ Berkeley Sensor and Actuator Center, University of California, Berkeley, California 94720, United States

*Corresponding Author: ajavey@ berkeley.edu 


\section{Calculation of the field effect mobility}

For calculating the field effect mobility for the device shown in Figure 5d, we used the following equation.

$\mu=L /\left(V_{\mathrm{DS}} C_{\mathrm{ox}} W\right) \times\left(\mathrm{d} I_{\mathrm{DS}} / \mathrm{d} V_{\mathrm{GS}}\right)$

where $L$ and $W$ are the channel length and width, respectively, $V_{\mathrm{DS}}$ was set at $-1 \mathrm{~V}$, and $C_{\mathrm{ox}}$ was calculated from using a gate dielectric stack of $\mathrm{SiO}_{2} /$ parylene $/ \mathrm{SiO}_{2}$ with a dielectric constant of 3.9 and thickness of $10 \mathrm{~nm}$ for each $\mathrm{SiO}_{2}$ layer and a dielectric constant of 3 and thickness of $700 \mathrm{~nm}$ for the parylene $C$ layer. The total capacitance is calculated to be $3.71 \times 10^{-9} \mathrm{~F} / \mathrm{cm}^{2}$. The transonductance $\left(\mathrm{d} I_{\mathrm{DS}} / \mathrm{d} V_{\mathrm{GS}}\right)$ was extracted from the $I_{\mathrm{DS}}-V_{\mathrm{GS}}$ curves shown in Figure $5 \mathrm{~d}$. From this, a mobility of $\sim 2 \mathrm{~cm}^{2} / \mathrm{V} \mathrm{s}$ is calculated.

\section{Detailed setup of the roll-to-roll process}

The length between supply and collection rollers is $\sim 1 \mathrm{~m}$. All the apparatus (rollers and baths) are well aligned beforehand on a line to feed the PET film smoothly. A syringe pump (Harvard apparatus) was attached to the PET film as the collection roller to maintain a constant feeding speed of $\sim 10 \mathrm{~cm} / \mathrm{min}$. It should be noted that the length of the CNT bath decides the maximum speed of the roll-to-roll deposition. In this setup, we used a total $10 \mathrm{~cm}$ long CNT bath (obtained from two 5 $\mathrm{cm}$ baths). For faster printing speeds, the CNT bath can be scaled up to longer lengths. The depth of the suspension was $\sim 2 \mathrm{~mm}$. To counteract evaporation of the CNT suspension, fresh CNT suspension (SC suspension) was periodically added to the baths. The PLL and DI water baths are also about $10 \mathrm{~cm}$ in length. Movie 1 depicts the operation of the roll-to-roll process. 
(a) SDBS

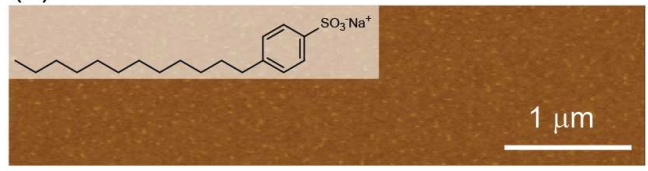

(b) SDS

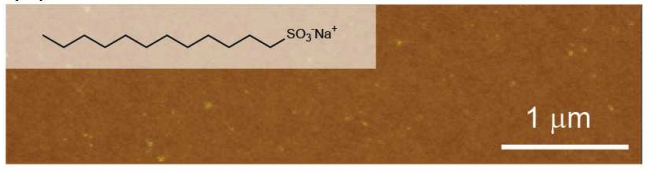

(c) SDC

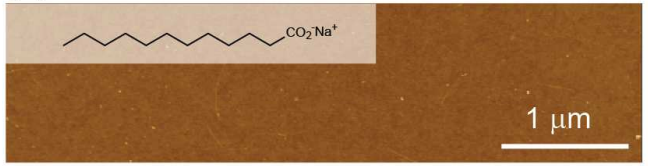

(d) SDBS/SC

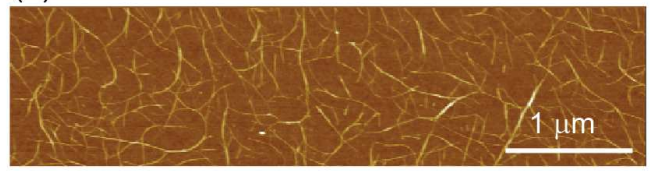

(e) SC in water

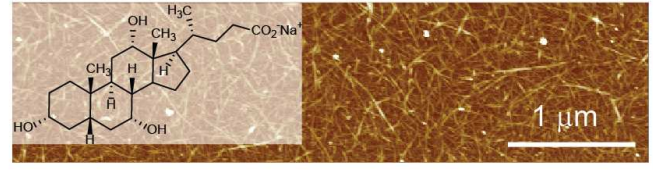

(f) SC in HEPES

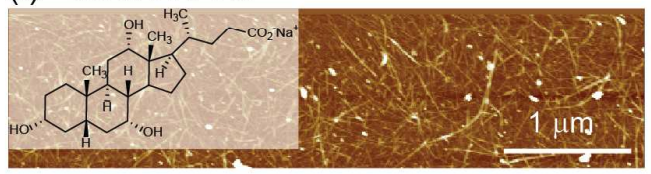

(g) SC in PBS

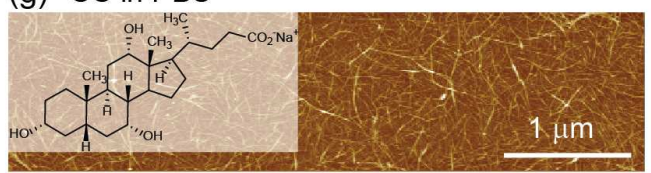

(h) SDeC in water

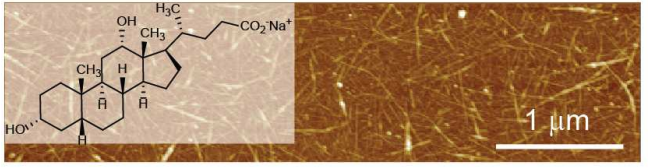

Figure S1 AFM images obtained from the deposition of various surfactant suspensions followed by washing with Milli-Q water and annealing at $200{ }^{\circ} \mathrm{C}$. The deposition time was $10 \mathrm{~min}$ at room temperature. Images are from (a) SDBS suspension $(12 \mathrm{mg} / \mathrm{ml})$, (b) SDS suspension $(10 \mathrm{mg} / \mathrm{ml})$, (c) SDC suspension $(7.5 \mathrm{mg} / \mathrm{ml})$, (d) SDBS/SC (12 mg/ml/ $15 \mathrm{mg} / \mathrm{ml}$ ) suspension, (e) SC suspension (15 mg/ml), (f) SC HEPES (40 mM) suspension (15 mg/ml), (g) SC PBS (2x) suspension (15 $\mathrm{mg} / \mathrm{ml})$ and (h) SDeC suspension (20 mg/ml). Milli-Q water was used as the solvent for making all suspensions. 


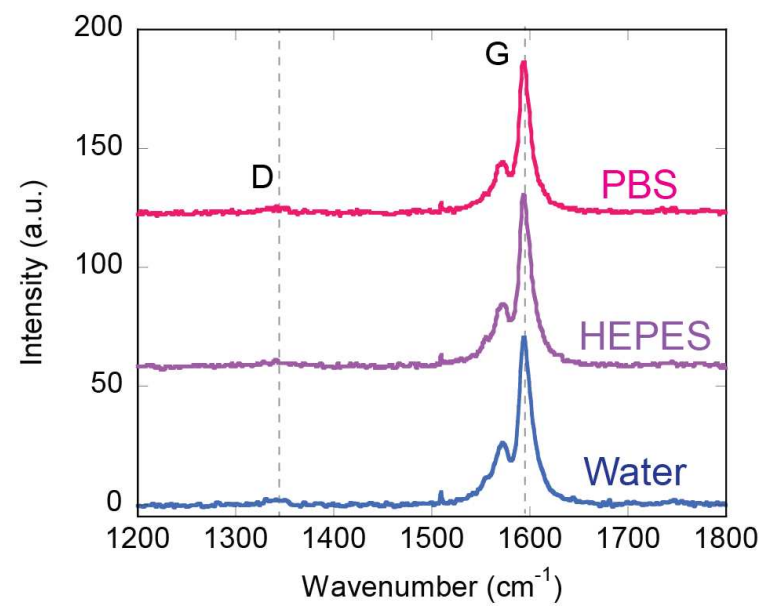

Figure S2 Raman spectra of the assembled CNTs on a PLL modified $\mathrm{SiO}_{2} / \mathrm{Si}$ wafer. CNTs are assembled from SC suspensions of (a) PBS (2x) buffer, (b) $40 \mathrm{mM}$ HEPES buffer and (c) Milli-Q water. $D$ (at $1350 \mathrm{~cm}^{-1}$ ) and $G$ (at $1600 \mathrm{~cm}^{-1}$ ) bands are labeled in the figure. The excitation wavelength was $532 \mathrm{~nm}$. 
(a) SDBS

(b) SDS

(c) SC in PBS

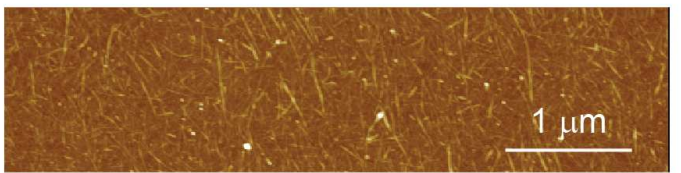

(d) SC in water

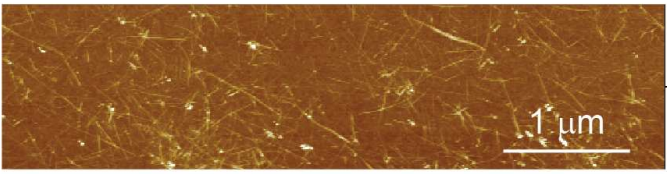

Figure S3 AFM images obtained from deposition of various CNT suspensions onto an APTES modified $\mathrm{SiO}_{2} / \mathrm{Si}$ wafer followed by Milli-Q washing and annealing at $200{ }^{\circ} \mathrm{C}$ in vacuum. The deposition time was $10 \mathrm{~min}$. APTES modification was done by $2 \mathrm{hr}$ immersion into an APTES ethanol solution $(100 \mathrm{mM})$ followed by washing with ethanol, then annealing at $100{ }^{\circ} \mathrm{C}$ for $1 \mathrm{~h}$ to remove any residual solvent. Images are obtained from CNT suspensions of (a) SDBS in Milli-Q water, (b) SDS in Milli-Q water, (c) SC in PBS buffer, and (d) SC in Milli-Q water. 
(a) Lipid dye (R18)

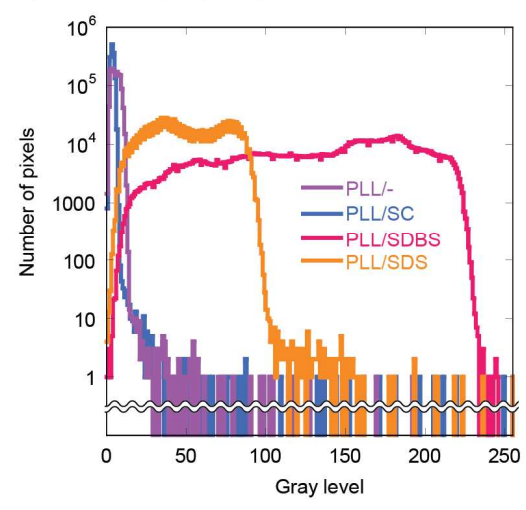

(b) Aromatic dye (perylene)

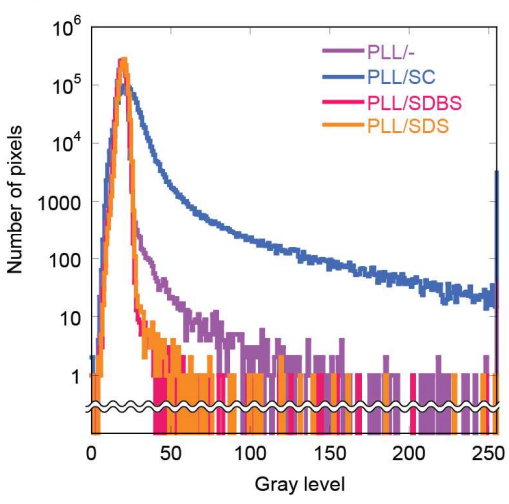

(c) Steroid dye

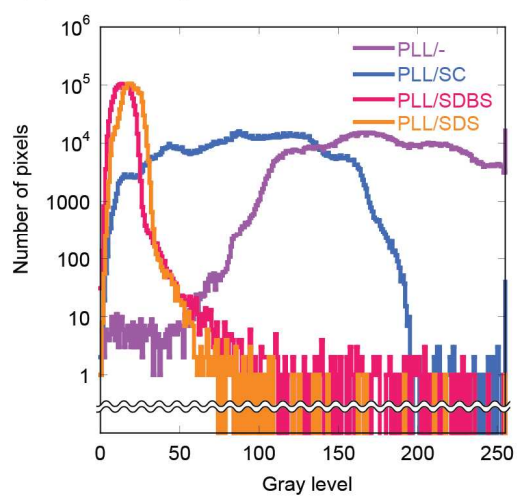

Figure S4 Number of pixels (counts in log scale) vs. intensity (gray level) curves obtained from the fluorescent microscope images shown in Figure 3a-c. The intensity range is from 0 to 255 . The graphs represent the (a) lipid dye (R18) shown in Figure 3a, (b) aromatic dye (perylene) shown in Figure 3b, and (c) steroid dye shown in Figure 3c. PLL/-, PLL/SC, PLL/SDBS, and PLL/SDS represent the process with no surfactant (Milli-Q water), SC, SDBS, and SDS surfactants, respectively.

(a) In Milli-Q water

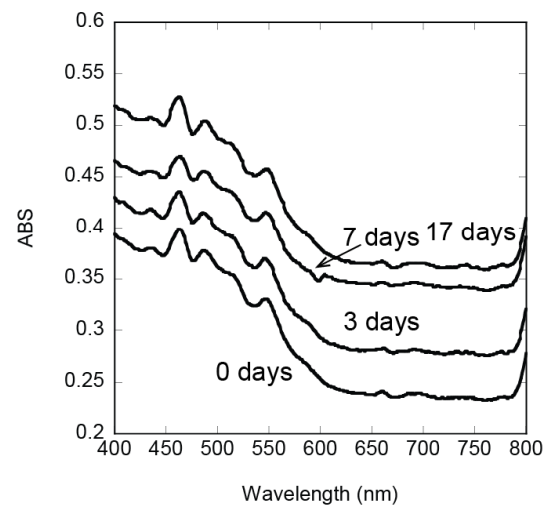

(b) In PBS solution

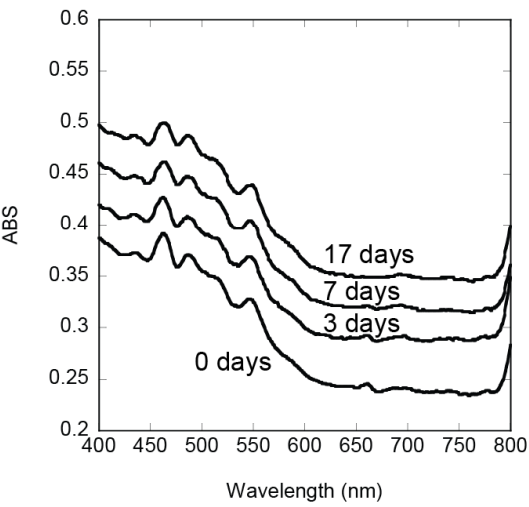

(c) In HEPES solution

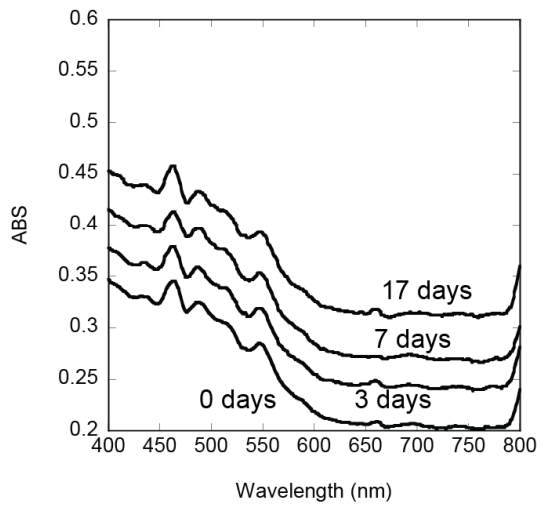

Figure S5 Optical spectra for CNT suspensions (SC surfactant) for up to 17 days from the date the solution was made to monitor stability. 0 days indicates the time the suspension was made. Graphs are obtained from CNT suspensions of (a) Milli-Q water, (b) PBS (2 x) buffer and (c) $40 \mathrm{mM}$ HEPES buffer. 
(a) 1 min deposition at r.t.

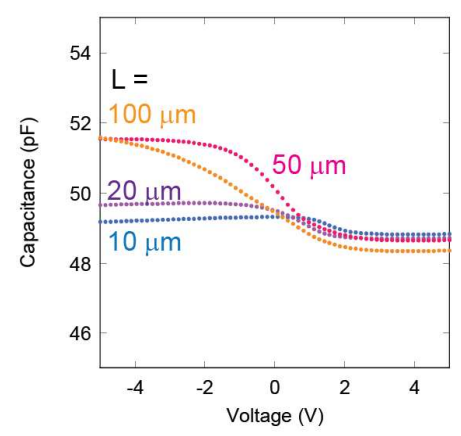

(b) 5 min deposition at r.t.

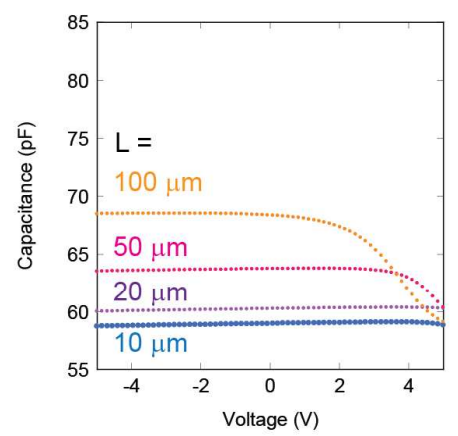

(c) 10 min deposition at r.t.

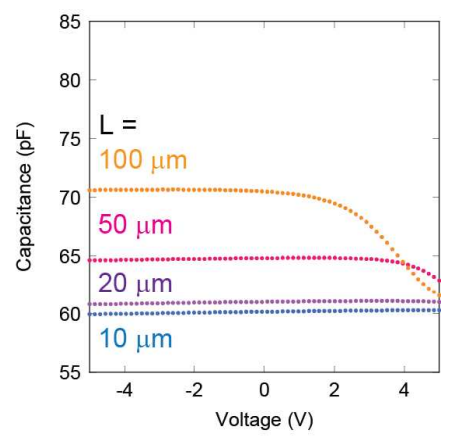

(d) 70 min deposition at r.t.

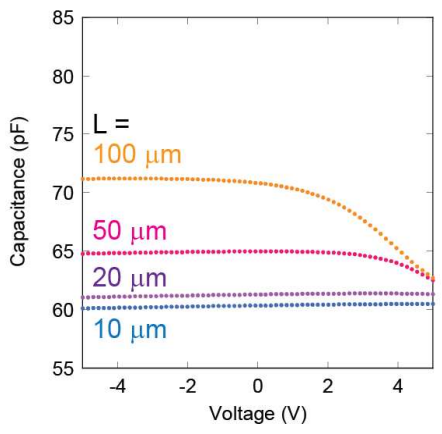

(e) 10 min deposition at 70 deg.C

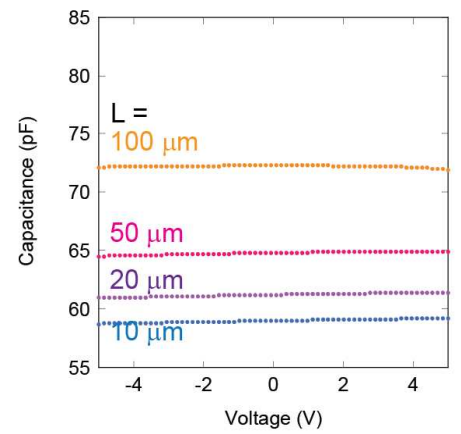

Figure S6 Capacitance vs. voltage $(C-V)$ data obtained from the setup shown in Figure 4a for various CNT deposition conditions on a PLL modified $\mathrm{SiO}_{2} / \mathrm{Si}$ wafer. Measurements are done with a $100 \mathrm{mV}$ a.c. signal at $100 \mathrm{kHz}$. Graphs are from (a) 1 min deposition at r.t., (b) 5 min deposition at r.t., (c) $10 \mathrm{~min}$ deposition at r.t., (d) $70 \mathrm{~min}$ deposition at r.t. and (e) $10 \mathrm{~min}$ deposition at $70{ }^{\circ} \mathrm{C}$. 


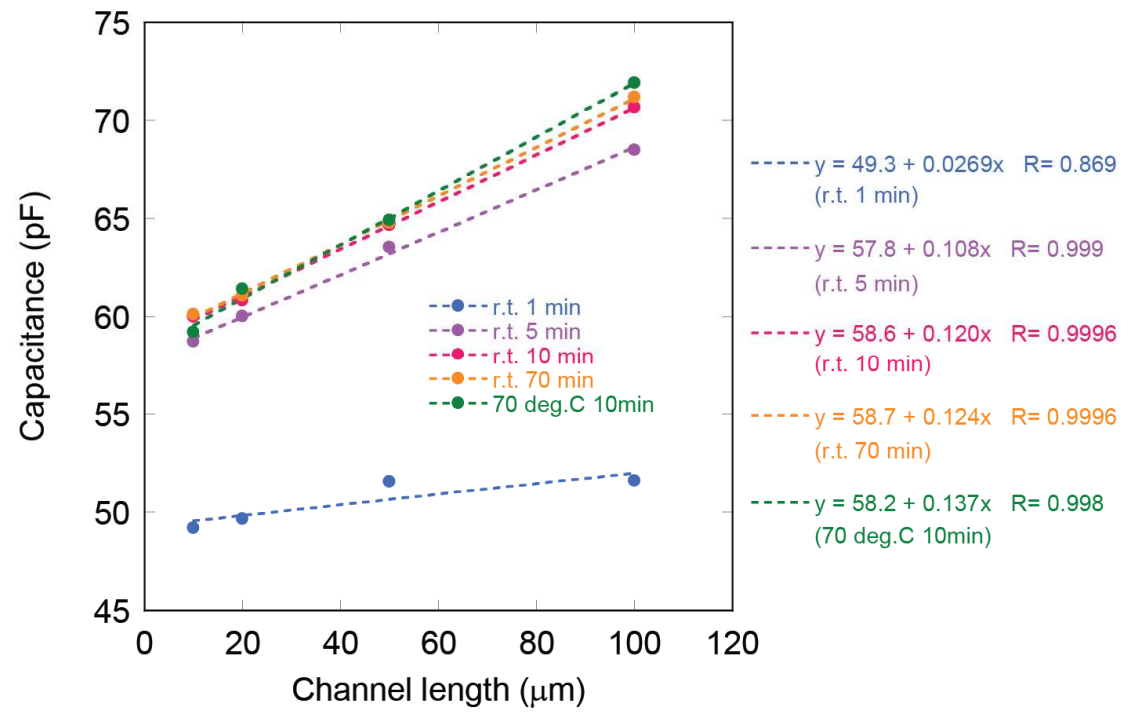

Figure S7 Capacitance vs. channel length plot obtained from $C$ - $V$ measurements shown in Figure S6. All plots show a good linear trend depending on the channel lengths. 
(a)

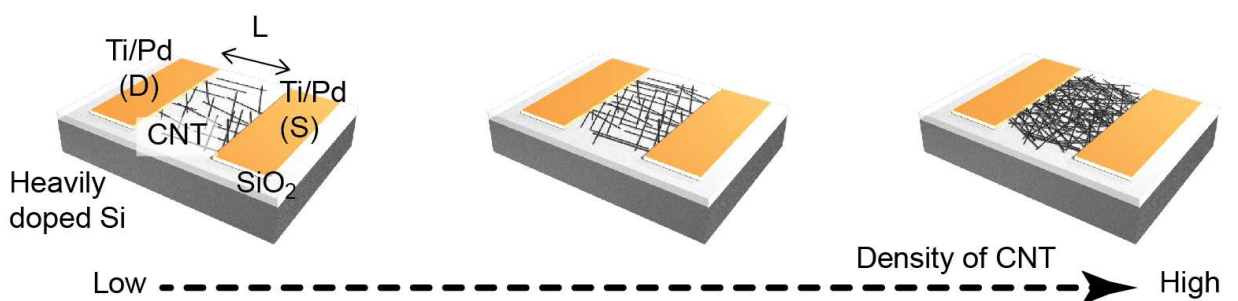

(b)
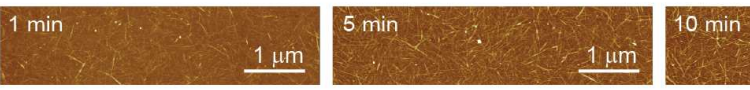

10 min

$-0---1$

(c)
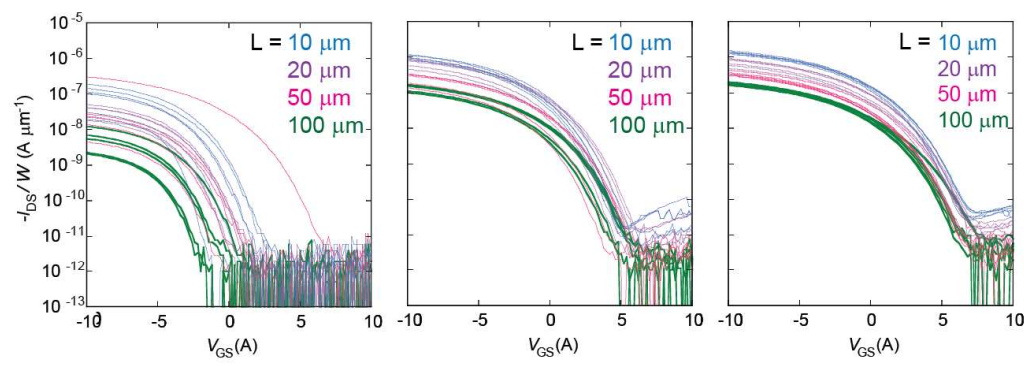

$70 \mathrm{~min}$

$$
\text { High }
$$

(d)

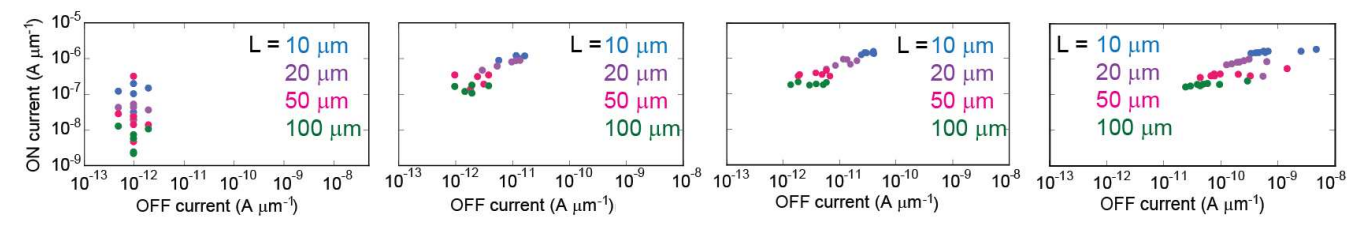

Figure S8 AFM images and drain current vs. gate voltage curves for various deposition times and channel lengths. CNT suspensions used are PBS buffered suspensions. The devices are made onto PLL modified $\mathrm{SiO}_{2} / p^{+}$-Si wafers. (a) Schematic illustration of the devices with different densities of CNTs. S and D electrodes are separated by CNT channels with length $L$. (b) AFM images of the assembled CNTs with various deposition times: 1, 5, 10, and 70 min from left to right. (c) Transfer characteristic curves of the assembled CNTs with various channel lengths $(L)$ from 10 to $100 \mu \mathrm{m}$. Widths $(W)$ are normalized (Actual $W$ used ranged from 50 to $200 \mu \mathrm{m}$ ). $V_{\mathrm{DS}}=-5 \mathrm{~V}$. The deposition times are 1, 5, 10, and 70 min from left to right. (d) Plots of on current vs. off current for the various channel lengths from 10 to $100 \mu \mathrm{m}$. The deposition times are 1, 5, 10, and $70 \mathrm{~min}$ from left to right. The graphs indicates the uniformity of on and off currents; 5-10 min deposition times show small variation between devices. 


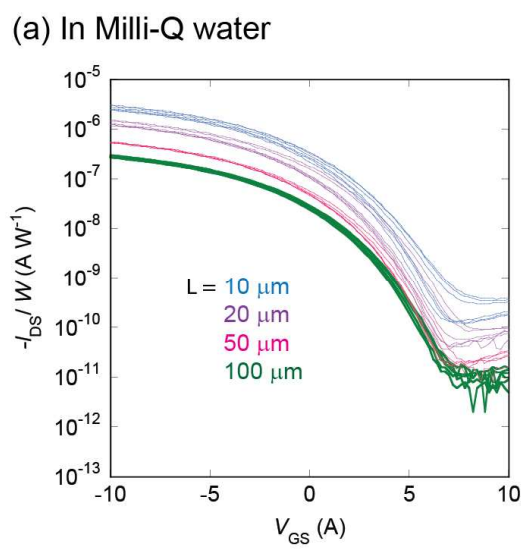

(b) In PBS solution

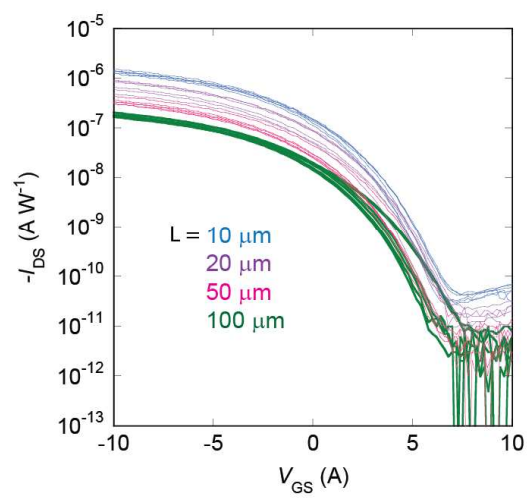

(c) In HEPES solution

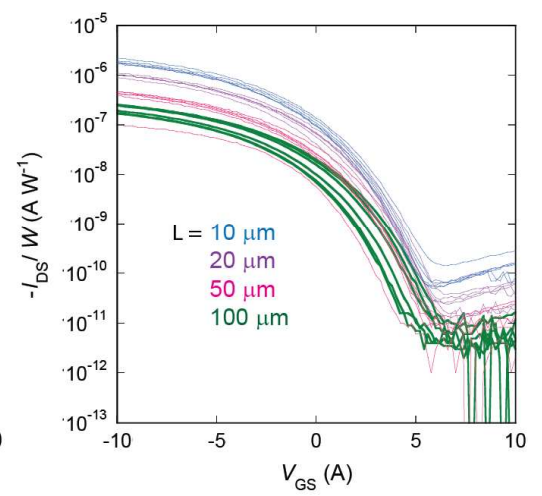

Figure S9 Drain current vs. gate voltage curves for CNT TFTs obtained from various CNT suspensions (SC surfactant): Suspensions are in (a) Milli-Q water, (b) PBS $(2 \times)$ buffered solution and (c) $40 \mathrm{mM}$ HEPES buffered solution. $V_{\mathrm{DS}}=-5 \mathrm{~V}$. Channel lengths $(L)$ are from 10 to $200 \mu \mathrm{m}$. Widths $(W)$ are normalized ( $W=50 \mu \mathrm{m}$ to $200 \mu \mathrm{m}$ ). The deposition time is $10 \mathrm{~min}$ in all cases. Device structures are the same as shown in Figure S8.

(a)

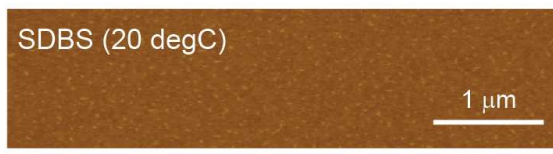

(b)

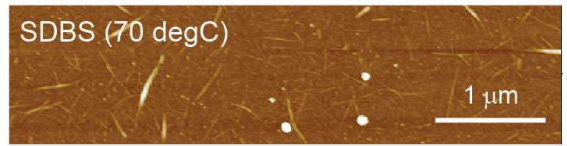

(c) $\mathrm{SC}(20 \mathrm{deg} \cdot \mathrm{C})$

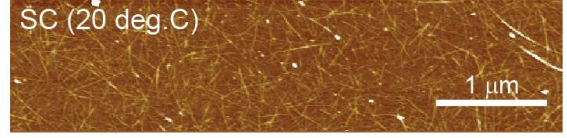

(d) Sc (70 dég c)

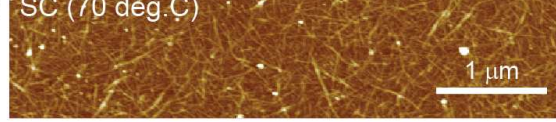

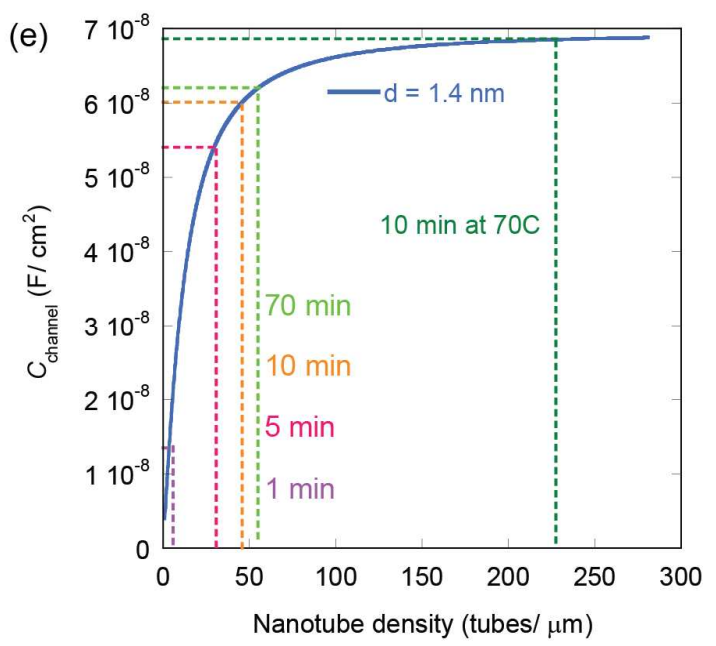

Figure S10 (a-d) AFM images of the assembled CNTs from suspensions with SDBS and SC surfactant onto PLL modified $\mathrm{SiO}_{2} / p^{+}-\mathrm{Si}$ wafers. The deposition times are $10 \mathrm{~min}$. Images are obtained from depositions of (a) SDBS suspension at $20^{\circ} \mathrm{C}$ and (b) $70{ }^{\circ} \mathrm{C}$, (c) SC suspension at $20{ }^{\circ} \mathrm{C}$ and $(\mathrm{d}) 70{ }^{\circ} \mathrm{C}$. (e) Capacitance $\left(C_{\text {channel }}\right)$ vs. tube density (tubes/ $\left.\mu \mathrm{m}\right)$ plots estimated from equation 2 in the text and the corresponding tube density for each deposition time. Tube density varies from 5 to 230 (tubes/ $\mu \mathrm{m}$ ) from $1 \mathrm{~min}$ deposition at r.t. to $10 \mathrm{~min}$ deposition at $70{ }^{\circ} \mathrm{C}$.

Movie 1 The setup and actual scene of the roll-to-roll process for CNT assembly. The movie is played at $6 \times$ real time. 